\title{
Classical Neurotransmitters and Neuropeptides Involved in Schizophrenia: How to Improve the Therapeutic Effect of the Antipsychotic Drugs
}

\author{
Felix-Martin Werner ${ }^{1,2}$ and Rafael Coveñas ${ }^{2}$ \\ 1. Higher Vocational School of Elderly Care and Occupational Therapy, Euro Academy Pößneck, Pößneck, Thuringia 07381, \\ Germany
}

2. Laboratory of Neuroanatomy of the Peptidergic Systems (Lab. 14), INCYL (Institute of Neurosciences of Castilla y León), University of Salamanca, Salamanca, Castilla-León 37007, Spain

\begin{abstract}
We describe the alterations of classical neurotransmitters and neuropeptides in schizophrenia. In this disease, susceptibility genes encode GABA (gamma-aminobutyric acid) and glutamate hypofunction and dopamine hyperactivity. A neural network is developed in the mesolimbic system, the hippocampus and the prefrontal cortex. According to this neural network, dopamine and serotonin hyperactivity might be due to a reduced presynaptic inhibition through GABAergic and glutaminergic neurons. A survey of the therapeutic and adverse effects of commonly prescribed and of recently developed second-generation antipsychotic drugs is given. The interaction with other specific subreceptors of classical neurotransmitters and neuropeptides is suggested to improve the antipsychotic effect. In the treatment of schizophrenia, pharmacotherapy should be combined with psychoeducation. Accordingly, a recurrence of psychotic symptoms could be prevented in a long-term treatment.
\end{abstract}

Key words: Acetylcholine, antipsychotic drugs, dopamine, cholecystokinin, GABA, glutamate, neurotensin, serotonin.

\section{Introduction}

Schizophrenia is a genetic mental illness that affects $1 \%$ of the population. Some susceptibility genes of the disease have been discovered [1]. Schizophrenia becomes manifest as an acute psychosis with positive symptoms such as paranoia, hallucinations, and illusions after a prodromal phase of about seven years with negative symptoms such as social withdrawal, mutism and altered emotional reaction $[1,2]$. It is a chronic disease with positive and negative symptoms and cognitive deficits [2]. In this review, we focus mainly our study on the paranoid and hallucinatory form of schizophrenia. In the mesolimbic system and the hippocampus, dopamine hyperactivity via D2 receptors occurs. Dopamine hyperactivity could be

Corresponding author: Felix-Martin Werner, M.D., research field: neural networks in neurological and psychiatric diseases. E-mail: felixm-werner@versanet.de. caused partly by a reduced presynaptic inhibition through GABAergic neurons, via GABAA receptors. Moreover, serotonin (5-HT) hyperactivity via 5-HT2A receptors occurs and this could be caused through a reduced presynaptic glutaminergic inhibition of the serotonergic neurons via NMDA (N-methyl-D-aspartate) receptors [3]. However, other classical neurotransmitters (e.g., acetylcholine) acting at specific subreceptors, adenosine, metabotropic glutaminergic receptors and nicotinic cholinergic receptors also play an important role in the pathophysiology of schizophrenia [4-6].

Some neuropeptides, for example, neurotensin, cholecystokinin and neuropeptide $\mathrm{Y}$ showed alterations in several brain areas of experimental animals, in which psychotic symptoms were induced experimentally [7-12]. NMDA antagonists induced schizophrenia-like symptoms that can be ameliorated 
by 5-HT2A antagonists, but not by D2 antagonists.

Here, a survey of some susceptiblity genes, such as catechol-O-methyl transferase, monoamine oxidase, glutamic acid decarboxylase 67, dysbindin-1 and neuregulin-1 is given. The coherence between the genetic localization and the cellular function is also indicated [3]. Neural networks are important in developing a multimodal pharmacotherapy and they have been developed in neurological and psychiatric diseases such as Alzheimer's and Parkinson's disease, generalized epilepsy and major depression [13, 14]. In this sense, in schizophrenia neural networks in the mesolimbic system, the hippocampus and the prefrontal cortex are here pointed out and we have also considered in these neuronal networks the interaction between classical neurotransmitters and neuropeptides [12].

Schizophrenia is treated by administering second-generation antipsychotic drugs which are mostly D2 and 5-HT2A antagonists. The therapeutic and adverse effects of commonly prescribed antipsychotic drugs and of recently developed antipsychotic drugs such as lurasidone cariprazine are discussed $[12,15]$. The interaction with other specific subreceptors others than D2 and 5-HT2A receptors is presentend in order to improve the antipsychotic effect [4, 6, 16, 17]. An additional psychoeducation and sociotherapy in order to deal better with the adverse effects and to reduce psychotic symptoms is considered. Thus, the purpose of this paper is to give hints to improve the treatment of schizophrenia and the care of schizophrenic patients in order to achieve a satisfactory long-term treatment.

\section{Alteration of Classical Neurotransmitters in Schizophrenia}

In schizophrenia, after the dopamine hypothesis was introduced in 1963, the serotonin and glutamate hypothesis was added [12]. In this disease, the alterations of classical neurotransmitters in the mesolimbic system, the hippocampus and the prefrontal cortex can be considered in a multi-neurotransmitter system [12]. In these brain regions, the alteration of neurotransmitters includes dopamine and serotonin hyperactivity and GABA and glutamate hypoactivity. Susceptibility genes, not only encoding dopamine hyperactivity like catechol-O-methyl transferase and monoamine oxidase, but also encoding GABA deficiency such as glutamic acid decarboxylase 67 and glutamate hypoactivity via NMDA receptors have been reported [13, 14, 18].

\subsection{Dopamine}

Dopamine hyperactivity occurs in the mesolimbic system and the hippocampus via $\mathrm{D}_{2}$ receptors (Fig. 1). Typical neuroleptics are $\mathrm{D}_{2}$ antagonists and reduce dopamine hyperactivity. Atypical neuroleptics or second-generation antipsychotic drugs are D2 and 5-HT2A antagonists and reduce dopamine and serotonin hyperactivity [15]. The antipsychotic drug aripiprazol is a partial D2 agonist and improves cognitive functions, but impairs motor activities [19]. Dopamine hyperactivity is associated with polymorphisms of the catechol-O-methyl transferase gene and reduced dopamine degradation leads to dopamine hyperactivity and cognitive impairments [20]. The action of the enantiomers (-)-OSU-6,162 and (+)-OSU-6,162 has been reported, which are partial agonists at both D2 and 5-HT2A receptors. In clinical trials, it should be examined whether OSU-6,162 exerts a sufficient antipsychotic effect or not [21].

\subsection{Serotonin}

In schizophrenia, 5-HT hyperactivity occurs in the mesolimbic system and the hippocampus (Fig. 1). The following question arises, whether 5-HT hyperactivity is encoded genetically or not. The 5-HT transporter gene has been examined in patients with psychotic and affective disorders showing a history of childhood trauma [22]. It was reported that carriers of the short allele had increased negative psychological reactions in comparison to those carrying the long allele [22]. 


\section{Brain centers involved in schizophrenia}

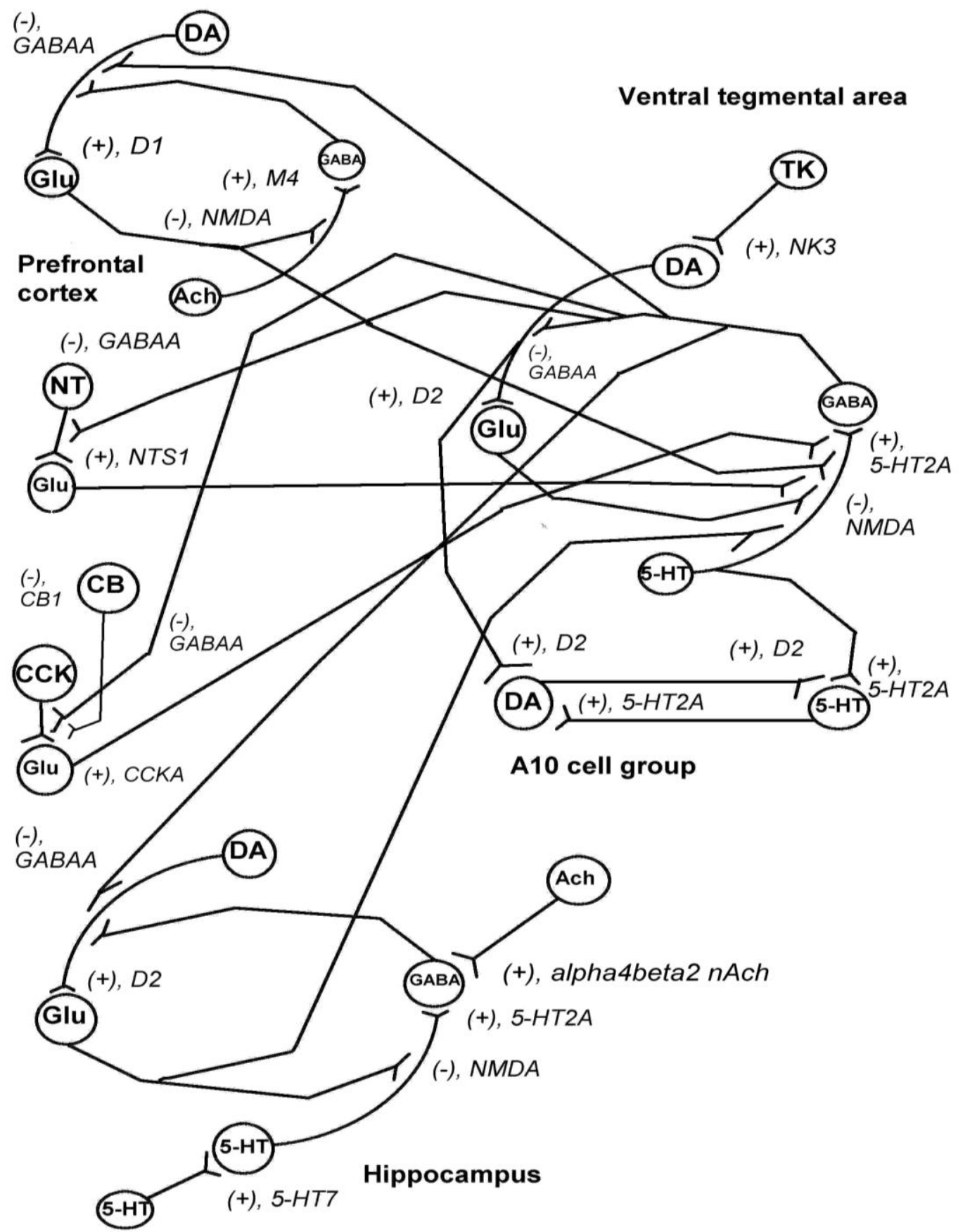

Fig. 1 Neuronal pathways, classical neurotransmitters and neuropeptides involved in schizophrenia.

5-HT: serotonin; Ach: acetylcholine; CB: cannabiniod; CCK: cholecystokinin; DA: dopamine; GABA: gamma-aminobutyric acid; Glu: glutamate; NT: neurotensin; TK: tachykinin. The following subreceptors are indicated: alpha4beta2nAch: a subreceptor of the nicotinic cholinergic receptor; CB1: a subreceptor of the cannabinoid receptor; CCKA: a subreceptor of the cholcystokinin receptor; D1: a subreceptor of the dopaminergic receptor; D2: a subreceptor of the dopaminergic receptor; GABAA: a subreceptor of the GABAergic receptor; 5-HT2A: a subreceptor of the serotonergic receptor; 5-HT7: a subreceptor of the serotonergic receptor; M4: a subreceptor of the muscarinic cholinergic receptor; NK3: a subreceptor of the tachykinin receptor; NMDA (N-methyl-D-aspartate) receptor: a subreceptor of the ionotropic glutaminergic receptor; NTS1: a subreceptor of the neurotensin receptor. A plus mark indicates a postsynaptic excitatory impulse; a minus mark indicates a presynaptic inhibitory impulse. 
In mice, schizophrenia-like behavior induced by MK-801, an NMDA antagonist, could be improved by alstonine, a D2 and 5-HT2A antagonist and by ritanserin, a 5-HT2A and 5-HT2C antagonist [23]. From this experiment, it can be concluded that a reduced glutaminergic presynaptic inhibition via NMDA receptors of 5-HT2A serotonergic neurons can induce 5-HT hyperactivity and this can be treated with 5-HT2A antagonists [23].

The newer antipsychotic drug cariprazine has a partial agonism at D2 and D3 receptors and a 5-HT1A agonistic effect [4]. It is known that antipsychotic drugs interfering with other subreceptors than the D2 receptors have therapeutic effects upon negative schizophrenic symptoms [4]. Olanzapine, a D2 and 5-HT2A antagonist with a great affinity for the 5-HT2A receptor has a superior therapeutic effect upon negative schizophrenic symptoms than other antipsychotic drugs [15].

\subsection{Gamma-aminobutyric Acid}

In the mesolimbic system and the hippocampus (Fig. 1), a decreased presynaptic GABAergic inhibition via $\mathrm{GABA}_{\mathrm{A}}$ receptors could contribute to dopamine hyperactivity via $\mathrm{D} 2$ receptors [12].

The concentration changes in dopamine, DOPAC (3,4-dihydroxyphenylacetic acid), glutamate and GABA were examined in the rat hippocampus and the mPFC (medial prefrontal cortex) in three animal groups: the first group received a single dose of MK-801, a NMDA antagonist; the second group was given a chronic administration of MK-801, and the third group acted as the control group [24]. In the first and second groups, the concentrations of dopamine, DOPAC, glutamate and GABA decreased in the hippocampus and the $\mathrm{mPFC}$, whereas in the control group the levels of these neurotransmitters were unchanged [24]. Besides, cognitive dysfunction in schizophrenia was found to be associated with dysfunction in the GABAergic neurotransmission. Neural circuits are linked to neural synchrony, e.g., oscillatory activities of the neurons are synchronized [25]. Consequently, drugs acting at GABAergic subreceptors could be novel candidate drugs in the treatment of schizophrenia [25]. GABAA agonists which interfere with the alpha1 or alpha2 subunit of the $\mathrm{GABA}_{\mathrm{A}}$ receptor could improve cognitive function or exert an anxiolytic effect [26].

\subsection{Glutamate}

Glutamate, a mainly excitotoxic, postsynaptic excitatory and partly presynaptic inhibitory neurotransmitter was found to have a dysfunction in schizophrenia in the mesolimbic system, the hippocampus and the PFC (Fig. 1) [3]. Glutamate acts on ionotropic glutaminergic receptors, i.e., NMDA, kainate and AMPA receptors and on metabotropic glutaminergic receptors [12]. Glutamate hypofunction at NMDA receptors is encoded by the susceptibility genes dysbindin-1 and neuregulin-1 [13]. In the mesolimbic system and the hippocampus, due to the susceptibility genes, glutaminergic neurons weakly inhibit via NMDA receptors 5-HT2A serotonergic neurons (Fig. 1) [12]. It has been reported in an animal model of schizophrenia that after administration of MK-801, the extracellular glutamate concentration was increased in the PFC and that the concentration was normalized after treatment with risperidone or paliperidone; that is after the treatment with D2 and 5-HT2A antagonists [27].

Agonists of mGluR (metabotropic glutaminergic receptors), for example mGlu2/3R agonists or m5GluR antagonists could be novel targets in the treatment of schizophrenia improving positive, negative and cognitive schizophrenic symptoms, because they normalize glutamate neurotransmission in the PFC [6].

\subsection{Acetylcholine}

Acetylcholine is a postsynaptic excitatory neurotransmitter that acts on nAchRs and MAchRs (nicotinic and muscarinic cholinergic receptors). 
Agonists of nicotinic (alpha7 and alpha2beta4) and muscarinic (M1 and M4) cholinergic receptors could be important in the treatment of the cognitive symptoms in schizophrenia [28]. In animal experiments, it has been shown that positive allosteric modulators of the alpha2beta4 nicotinic cholinergic receptors improved cognitive functions [29]. A positive allosteric modulator of alpha7 nicotinic cholinergic receptors also improved cognitive processes [30]. In the section about neural circuits, it will be shown that in the hippocampus nicotinic cholinergic neurons activate the GABAergic neurotransmission (Fig. 1).

$\mathrm{M}_{1}$ or $\mathrm{M}_{4}$ agonists could be of therapeutic benefit in the treatment of schizophrenia. In the PFC, an antagonistic interaction between D1 dopaminergic and M4 muscarinic cholinergic neurons occurs (Fig. 1). Consequently, $\mathrm{M}_{4}$ agonists could reduce D1 dopamine hyperactivity through an increased presnaptic GABAergic inhibition of D1 dopaminergic neurons, via $\mathrm{GABA}_{\mathrm{A}}$ receptors [31].

\section{Alteration of Neuropeptides in Schizophrenia}

Here, in schizophrenia, alterations in the level of neuropeptides in the mesolimbic system, the hippocampus and the PFC will be described (Fig. 1) [7, 9-12]. Besides, the following issue will be addressed, whether analogues, agonists or antagonists of neuropeptides could be used as an additional therapy in schizophrenia [12].

\subsection{Cholecystokinin}

CCK (cholecystokinin) concentrations were found to be decreased in the striatum, the nucleus accumbens, the amygdala and the frontal and temporal cortices in drug-free schizophrenic patients, whereas the concentration of CCK was augmented in the striatum and in the frontal and temporal cortices after neuroleptic treatment $[32,33]$. There is a relationship between CB1 cannbinoid receptors and CCK immunoreactive neurons in the dorsolateral PFC [16]. In the section about neural circuits, it will be pointed out that cannbinoid neurons strongly inhibit, via CB1 receptors, CCK neurons in the dorsolateral PFC (Fig. 1) [16].

An association between the CCKA gene and persistant auditory hallucination has been reported [17]. The following question arises, whether CCKA agonists could be of therapeutic value in the treatment of persistant auditory hallucinations [17].

\subsection{Neurotensin}

Neurotensin is one of the most essential neuropeptides in schizophrenia, because it acts as an endogeneous neuroleptic. Reduced neurotensin levels were found in the cerebrospinal fluid of schizophrenic patients, in the mesolimbic system and in the PFC; these levels were normalized after neuroleptic treatment [34]. It is known that NTS1 receptor agonists show antipsychotic properties [35] and that neurotensin neurons in the PFC are connected to serotonergic neurons in the mesolimbic system through glutaminergic neurons (Fig. 1) [36]. Moreover, it has been reported that NT69L, a neurotensin analogue, showed antipsychotic effects in an animal model of schizophrenia [37].

\subsection{Tachykinins (Substance P)}

Tachykinins (substance $\mathrm{P}$, neurokinin $\mathrm{A}$ and $\mathrm{B}$ ) exert their physiological actions via three receptors: NK1, NK2 and NK3. While substance P is important in major depression, neurokinin $\mathrm{A}$ and $\mathrm{B}$ play an important role in the pathogenensis of schizophrenia [38]. RO-458,328, a novel NK1 and NK3 receptor antagonist could be beneficial in the treatment of schizophrenia, because an antagonism at NK3 receptors reduced the firing rate of dopaminergic neurons in the mesolimbic system (Fig. 1) [39, 40].

\section{Susceptibility Genes in Schizophrenia}

After the development of new genetic techniques, 
some susceptibility genes in schizophrenia have been discovered [41]. The risk genes neuregulin-1, dysbindin-1, catechol-O-methyltransferase, monamine oxidase and glutamic acid decarboxylase 67 will be considered [41].

\subsection{Neuregulin-1}

Some haplotypes of the neuregulin-1 gene have been described as a risk gene for schizophrenia [42]. The neuregulin-1 gene regulates neurotransmitter receptor expression, including the subreceptor NMDA $2 \mathrm{C}$ and the GABA receptor beta unit. Because neuregulin-1 transcript expression is reduced, the haplotypes of the neuregulin-1 gene are linked to glutamate hypoactivity via NMDA receptors [42].

\subsection{Dysbindin-1}

Reduced mRNA dysbindin-1 levels were found in the hippocampus of schizophrenic patients [43]. It has been reported that the haplotype of the dysbindin-1 gene was correlated with the form and severity of the psychosis [44]. Haplotypes of the dysbindin-1 gene are linked to glutaminergic dysfunctions in the hippocampal formation [43].

\subsection{COMT (Catechol-O-metyltransferase)}

Some haplotypes of the COMT gene are a risk for schizophrenia. These haplotypes are associated to a decreased activity of the COMT enzyme. This enzyme catalyzes the breakdown of dopamine. Due to the reduced activity of this enzyme, dopamine hyperactivity occurs in the mesolimbic system and the hippocampus [13].

\subsection{MAO (Monoamine Oxidase)}

The isoenzymes MAO-A and MAO-B catalyze the metabolism of dopamine [45]. Some haplotypes of the MAO enzyme have been discovered in schizophrenic patients and they have been correlated with a decreased degradation of dopamine [45].

\subsection{GAD (Glutamic Acid Decarboxylase) 67}

GAD 67 has also been described as a susceptibility gene for schizophrenia with descreased concentrations of the presynaptic neurotransmitter GABA, which exerts an inhibitory effect on the projections to the PFC $[46,47]$.

\section{Schizophrenia: Form the Genetic Localization to the Cellular Mechanism}

The risk genes dysbindin-1, neuregulin-1 and GAD 67 are linked to a glutamate and GABA hypofunction in the hippocampus and the mesolimbic system (Fig. 1) [3]. The risk genes COMT and MAO-B are associated with a decreased dopamine breakdown and hence with a dopamine hyperactivity in the above-mentioned brain regions (Fig. 1) [3]. NMDA antagonists can induce schizophrenia-like symptoms which can be relieved by 5-HT2A antagonists [3]. The possible neural networks involved in schizophrenia could be described as follows (Fig. 1): in the mesolimbic system, due to the genes dysbindin-1 and neuregulin-1, glutaminergic neurons weakly inhibit presynaptically 5-HT2A serotonergic neurons which show a high activity. The serotonergic neurons are connected to GABAergic neurons. Due to the GAD 67 gene, GABAergic neurons weakly inhibit presynaptically D2 dopaminergic neurons which show a high activity. Tachykinin neurons activate, via NK3 receptors, dopaminergic neurons. Dopamine hyperactivity is enhanced through the reduced activity of the COMT and MAO-B enzymes which leads to a reduced degradation of dopamine [3]. In the A10 cell group, dopaminergic and serotonergic neurons activate each other through D2 and 5-HT2A receptors enhancing dopamine and serotonin hyperactivity.

GABAergic neurons send projections to neurotensin neurons located in the prefrontal cortex, which transmit a weak activating impulse to glutaminergic neurons via NTS1 receptors. GABAergic neurons are connected as well to cholecystokinin neurons located in the prefrontal 
cortex, which weakly activate glutaminergic neurons via CCKA receptors. The glutaminergic neurons inhibit serotonergic neurons in the mesolimbic system. Cannabinoid neurons inhibit CCK neurons via CB1 receptors. In the mesolimbic system, GABAergic neurons weakly inhibit, via GABAA receptors, D1 dopaminergic neurons in the prefrontal cortex which show a high activity. D1 dopaminergic neurons are connected to glutaminergic neurons which strongly inhibit, via NMDA receptors, M4 muscarinic cholinergic neurons. The latter neurons are connected to GABAergic neurons. The glutaminergic neurons located in the prefrontal cortex weakly inhibit, via NMDA receptors, serotonergic neurons in the mesolimbic system.

GABAergic neurons located in the mesolimbic system weakly inhibit, via GABAA receptors, D2 dopaminergic neurons in the hippocampus which have a high activity. The D2 dopaminergic neurons are connected to glutaminergic neurons which weakly inhibit, via NMDA receptors, 5-HT2A serotoninergic neurons which have a high activity. The 5-HT2A serotonergic neurons are connected to GABAergic neurons, while nicotinic cholinergic neurons activate GABAergic neurons via alpha4beta2 nAch receptors. Serotonergic neurons with a high activity are activated through other serotonergic neurons via 5-HT7 receptors. The glutaminergic neurons in the hippocampus, via NMDA receptors, weakly inhibit the serotonergic neurons located in the mesolimbic system.

\section{Second-Generation Antipsychotic Drugs}

In this section, conventional and some recently developed second-generation antipsychotic drugs are discussed. These antipsychotic drugs, which mostly are D2 and 5-HT2A antagonists, cause less seldom EPS (extrapyramidal symptoms) than first-generation antipsychotic drugs such as haloperidol, which are $\mathrm{D}_{2}$ antagonists $[12,15]$. The therapeutic effects on positive and negative schizophrenic symptoms as well as the adverse effects of these drugs will be also pointed out.

\subsection{Risperidone}

Risperidone is a D2 and 5-HT2A antagonist with a great affinity for the $\mathrm{D} 2$ receptor. As a consequence of its mechanism of action, risperidone causes more often EPS than olanzapine and quetiapine. Risperidone has a therapeutic effect also on negative schizophrenic symptoms, but to the same extent like olanzapine. It causes hyperprolactinemia, but less often than haloperidol [15]. A PET study revealed that the antipsychotic effect is reached, if the D2 receptor occupancy is between $65 \%-70 \%$ [48].

\subsection{Olanzapine}

Olanzapine is a D2 and 5-HT2A antagonist with a greater affinity for the 5-HT2A receptor than risperidone. Therefore, it causes less seldom EPS than risperidone and exerts a better therapeutic effect on negative schizophrenic symptoms than risperidone. Among these adverse effects are the following: weight gain, insulin resistance and increase of glucose [15, 48].

\subsection{Quetiapine}

Quetiapine is also a D2 and 5-HT2A antagonist with an even greater affinity for the 5-HT2A receptor than olanzapine. It has a slight tendency to cause EPS and has a comparable antipsychotic effect to other antipsychotic drugs [15]. Quetiapine causes increase of glucose and cholesterol to a smaller extent than olanzapine and it is a prolactin-sparing antipsychotic drug $[15,48]$.

\subsection{Ziprasidone}

Ziprasidone has a D2 and 5-HT2A antagonistic effect and an additional antidepressant effect through a 5-HT1A agonism. It has comparable therapeutic effects on positive and negative schizophrenic symptoms like other second-generation antipsychotic drugs. The occurrence of EPS is reduced [48]. The metabolic side effects are comparable to those 
induced by olanzapine [15].

\subsection{Clozapine}

Clozapine has a D3, D4 and 5-HT2A antagonistic effect. It is known that $10 \%-30 \%$ of the schizophrenic patients do not respond to an antipsychotic therapy. In most of these patients, clozapine can ameliorate the psychotic symptoms. Clozapine has a superior antipsychotic effect than other antipsychotic drugs and seldom causes EPS, because it does not interfere with the D2 receptor. In 3\% of the patients treated with clozapine neutropenia occurred, and in $0.8 \%$ of the patients, agranulocytosis appeared [48]. Clozapine can cause insulin resistance like olanzapine [15].

\subsection{Aripiprazole}

Aripiprazole has a partial D2 agonism effect, a 5-HT2A antagonistic effect and a 5-HT1A agonistic effect. It has comparable antipsychotic effects and it less often causes weight gain and EPS than other antipsychotic drugs. It has an additional effect in major depression and dementia, but the antipsychotic effect in demented patients is less efficient than when patients were treated with risperidone [48].

\subsection{Asenapine}

Asenapine is an antipsychotic drug with D2 and 5-HT2A antagonistic properties. It exerts a good antipsychotic effect and improves cognitive function. This effect was proved in an animal model of schizophrenia. The administration of asenapine improved the cognitive function and increased the dopamine and serotonin turnover in some rain regions [49].

\subsection{Lurasidone}

Lurasidone is an antipsychotic drug with D2, 5-HT2A and 5-HT7 antagonistic effect and also exerts an antidepressant effect through a 5-HT1A agonism. It has good therapeutic effects on positive and negative schizophrenic symptoms and improves cognitive function as a consequence of the 5-HT7 antagonistic effect [50].

\subsection{Cariprazine}

Cariprazine has a partial agonism at D2 and D3 receptors and has an antidepressant effect through a 5-HT1A agonism. It has comparable antipsychotic effects like other antipsychotic drugs. It enables a good quality of life. It remains to be examined whether cariprazine has a safe therapeutic effect in a long-term antipsychotic treatment [4].

\subsection{OSU-6,162}

OSU-6,162 is a novel antipsychotic drug which has a therapeutic effect not only in schizophrenia, but also in Parkinson's and Huntington's disease. It exerts a partial agonism at D2 and 5-HT2A receptors. It should be examined in clinical trials whether OSU-6,162 exerts a safe antipsychotic effect [21].

\section{Conclusion}

Once the patients have developed an acute psychosis, treatment with second-generation antipsychotic drugs is necessary. The commonly prescribed SGAs (second-generation antipsychotics) are risperidone, olanzapine, quetiapine, aripriprazole and clozapine [1]. In clinical trials, it should be examined whether positive and negative psychotic symptoms occur again in a long-term treatment of the disease. Besides, it should be of importance to compare two groups of patients: one group treated with SGA's and the second group treated with SGA's and receiving a supporting psychoeducation and sociotherapy [5]. A reason for recurrence of psychotic symptoms is the lack of patients's compliance, i.e., that the patients decide on their own to take no longer the antipsychotic drug. Psychoeducation can be a tool for the patients to deal with the side effects and can improve patients' compliance. Besides, the patients learn to differentiate between side effects and psychotic symptoms. They can learn a better inside in 


\section{How to Improve the Therapeutic Effect of the Antipsychotic Drugs}

the disease. It should be important to examine the risk genes for schizophrenia in a large cohort of patients. In that way, the treating physician would have a method to choose the appropriate antipsychotic drug. A schizophrenic patient having the COMT and MAO-B genes with a dopamine hyperactivity might profit from the SGA risperidone which shows a great affinity for the D2 receptor [3]. Nevertheless, it is essential to examine antipsychotic drugs interacting with other specific subreceptors than D2 and 5-HT2A receptors, for example GABAA, NMDA, nicotinic cholinergic and metabotropic glutaminergic receptors. Moreover, it should be investigated whether CCKA agonists could ameliorate persistent auditory hallucinations.

\section{References}

[1] Klosterkötter, J. 2008. "Indicated Prevention of Schizophrenia." Deutsches Ärzteblatt International 105 (30): 532-9.

[2] Huber, G., and Gross, G. 1989. "The Concept of Basic Symptoms in Schizophrenic and Schizoaffective Psychoses." Recenti Progressi in Medicina 80 (12): 646-52.

[3] Werner, F. M. 2006. "Schizophrenia: From the Genetic Localization to the Cellular Mechanism." Klinische Neurophysiologie 37: 19-20.

[4] Seneca, N., Finnema, S. J., Laszlovszky, I., Kiss, B., Horváth, A., Pásztor, G., Kapás, M., Gyertyán, I., Farkas, S., Innis, R. B., Halldin, C., and Gulyás, B. 2011. "Occupancy of Dopamine $\mathrm{D}(2)$ and $\mathrm{D}(3)$ and Serotonin 5-HT(1A) Receptors by the Novel Antipsychotic Drug Candidate, Cariprazine (RGH-188), in Monkey Brain Measured Using Positron Emission Tomography." Psychopharmacology 218 (3): 579-87.

[5] Horiguchi, M., Huang, M., and Meltzer, H. Y. 2011. "The Role of 5-hydroxytryptamine 7 Receptors in the Phencyclidine-Induced Novel Object Recognition Deficit in Rats." The Journal of Pharmacology and Experimental Therapeutics 338 (2): 605-14.

[6] Vinson, P. N., and Conn, P. J. 2011. "Metabotropic Glutamate Receptors as Therapeutic Targets for Schizophrenia.” Neuropharmacology 62 (3): 1461-72.

[7] Werner, F. M., and Coveñas, R. 2005. "Neuropeptides Involved in Schizophrenia." Current Topics in Neurochemistry 4: 35-49.

[8] Werner, F. M., and Coveñas, R. 2005. "Classical Neurotransmitters and Neuropeptides Involved in
Parkinson's Disease." Current Topics in Peptide and Protein Research 7: 75-84.

[9] Werner, F. M., and Coveñas, R. 2007. "Classical Neurotransmitters and Neuropeptides Involved in Parkinson and Alzheimer Diseases." In Focus on Neuropetide Research, edited by Coveñas, R., Mangas, A., and Nárvaez, J. A. Trivandrum: Transworld Research Network.

[10] Werner, F. M., and Coveñas, R. 2010. "Classical Neurotransmitters and Neuropeptides Involved in Major Depression: A Review." International Journal of Neuroscience 120 (7): 455-70.

[11] Werner, F. M., and Coveñas, R. 2011. "Classical Neurotransmitters and Neuropeptides Involved in Generalized Epilepsy: A Focus on Antiepileptic Drugs.” Current Medicinal Chemistry 18 (32): 4933-48.

[12] Werner, F. M., and Coveñas, R. 2013. "Classical Neurotransmitters and Neuropeptides Involved in Schizophrenia: How to Choose the Appropriate Antipsychotic Drug?" Current Drug Therapy 8 (2): 132-43.

[13] Collier, D. A., and Li, T. 2003. "The Genetics of Schizophrenia: Glutamate not Dopamine?" European Journal of Pharmacology 480 (1-3): 177-84.

[14] Weickert, C. S., Straub, R. E., McClintock, B. W., Matsumoto, M., Hashimoto, R., Hyde, T. M., Herman, M. M., Weinberger, D. R., and Kleinman, J. E. 2004. "Human Dysbindin (DTNBP1) Gene Expression in Normal Brain and in Schizophrenic Prefrontal Cortex and Midbrain." Archives of General Psychiatry 61 (6): 544-55.

[15] Werner, F. M., and Coveñas, R. 2014. "Safety of Antipsychotic Drugs: Focus on the Therapeutic and Adverse Effects." Expert Opinion on Drug Safety 13 (8): 1031-42.

[16] Eggan, S. M., Melchitzky, D. S., Sesack, S. R., Fish, K. N., and Lewis, D. A. 2010. "Relationship of Cannbinoid CB1 Receptor and Cholecystokinin Immunoreactivity in Monkey Dorsolateral Prefrontal Cortex." Neuroscience 169 (4): 1652-61.

[17] Sanjuan, J., Toirac, I., González, J. C., Leal, C., Moltó, M. D., Nájera, C., and De Frutos, R. 2004. "A Possible Association between the CCK-AR Gene and Persistent Auditory Hallucinatons in Schizophrenia." European Psychiatry 19 (6): 349-53.

[18] Nilsson, M., Waters, S., Waters, N., Carlsson, A., and Carlsson, M. L. 2001. “A Behavioural Pattern Analysis of Hypoglutaminergic Mice-Effects of Four Different Antipsychotic Agents." Journal of Neural Transmission 108 (10): 1181-96.

[19] Picada, J. N., Dos Santos Bde, J., Celso, F., Monteiro, J. D., Da Rosa, K. M., Camacho, L. R., Vieira, L. R., Freitas, T. M., Da Silva, T. G., Pontes, V. M., and Pereira, 
P. 2011. "Neurobehavioral and Genotoxic Parameters of Antipsychotic Agent Arpiprazole in Mice." Acta Pharmacologica Sinica 32 (10): 1225-32.

[20] Gupta, M., Kaur, H., Jajodia, A., Jain, S., Satyamoorthy, K., Mukerji, M., Thirthalli, J., Indian Genome Variation Consortium, and Kukreti, R. 2011. "Diverse Facets of COMT: From a Plausible Predictive Marker to a Potential Drug Target for Schizophrenia." Current Molecular Medicine 11 (9): 732-43.

[21] Burstein, E. S., Carlsson, M. L., Owens, M., Ma, J. N., Schiffer, H. H., Carlsson, A., and Hacksell, U. 2011. "II. In vitro Evidence that (-)-OSU6162 and (+)-OSU6162 Produce Their Behavioral Effects through 5-HT2A Serotonin and D2 Dopamine Receptors." Journal of Neural Transmission 118 (11): 1523-33.

[22] Aas, M., Djurovic, S., Athanasiu, L., Steen, N. E., Agartz, I., Lorentzen, S., Sundet, K., Andreassen, O. A., and Melle, I. 2011. "Serotonin Transporter Gene Polymorphism, Childhood Trauma, and Cognition in Patients with Psychotic Disorders." Schizophrenia Bulletin 38 (1): 15-22.

[23] Linck, V. M., Bessa, M. M., Herrmann, A. P., Iwu, M. M., Okunji, C. O., and Elisabetsky, E. 2012. "5-HT(2 A/C) Receptors Mediate the Antipsychotic-like Effect of Alstonine." Progress in Neuropsychopharmacology and Biological Psychiatry 36 (1): 15-22.

[24] Liu, Y., Tang, Y., Pu, W., Zhang, X., and Zhao, J. 2011. "Concentration Change of DA, DOPAC, Glu and GABA in Brain Tissues in Schizophrenia Developmental Model Rats Induced by MK-801." Zhong Nan Da Xue Bao Yi Xue Ban 36 (8): 712-9.

[25] González-Burgos, G., Fish, K. N., and Lewis, D. A. 2011. "GABA Neuron Alterations, Cortical Circuit Dysfunction and Cognitive Deficits in Schizophrenia." Neural Plasticity 2011: 723184.

[26] Atack, J. R. 2010. "GABAA Receptor Alpha2/Alpha3 Subtype-Selective Modulators as Potential Nonsedating Anxiolytics." Current Topics in Behavioral Neurosciences 2: 231-60.

[27] Roenker, N. L., Gudelsky, G., Ahlbrand, R., Bronson, S. L., Kern, J. R., Waterman, H., and Richtand, N. M. 2011. "Effect of Paliperidone and Risperidone on Extracellular Glutamate in the Prefrontal Cortex of Rats Exposed to Prenatal Immune Activation or MK-801." Neuroscience Letters 500 (3): 167-71.

[28] Jones, C. K., Byun, N., and Bubser, M. 2012. "Muscarinic and Nicotinic Acetylcholine Receptor Agonists and Allosteric Modulators for the Treatment of Schizophrenia." Neuropsychopharmacology 37 (1): 16-42.

[29] Timmermann, D. B., Sandager-Nielsen, K., Dyhring, T., Smith, M., Jacobsen, A-M , Nielsen, E., Grunnet, M., Christensen, J. K., Peters, D., Kohlhaas, K., Olsen, G. M., and Ahring, P. K. 2012. "Augmentation of Cognitive Function by NS9283, a Stochiometry-Dependent Positive Allosteric Modulator of Alpha2- and Alpha4-Containing Nicotinic Acetylcholine Receptors." British Journal of Pharmacology 167 (1): 164-82.

[30] Wallace, T. L., and Porter, R. H. 2011. "Targeting the Nicotinic Alpha7 Acetylcholine Receptor to Enhance Cognition in Disease.” Biochemical Pharmacology 82 (8): 891-903.

[31] Dencker, D., Wörtwein, G., Weikop, P., Jeon, J., Thomsen, M., Sager, T. N., Mork, A., Woldbye, D. P., Wess, J., and Fink-Jensen, A. 2011. "Involvement of a Subpopulation of Neuronal M4 Muscarinic Acetylcholine Receptors in the Antipsychotic-Like Effects of the M1/M4 Preferring Muscarinic Receptor Agonist Xanomeline.” Journal of Neuroscience 31 (16): 5905-8.

[32] Beinfeld, M. C., and Garver, D. L. 1991. "Concentration of Cholecystokinin in Cerebrospinal Fluid Is Decreased in Psychosis: Relationships to Symptoms and Drug Response." Progress in Neuropsychopharmacol and Biological Psychiatry 15 (5): 961-9.

[33] O'Connor, W. T. 2001. "Functional Neuroanatomy of the Ventral Striopallidal GABA Pathway. New Sites of Intervention in the Treatment of Schizophrenia." Journal of Neuroscience Methods 109 (1): 31-9.

[34] Cáceda, R., Kinkead, B., and Nemeroff, C. B. 2003. "Do Neurotensin Receptor Agonists Represent a Novel Class of Antipsychotic Drugs?" Seminars in Clinical Neuropsychiatry 8 (2): 94-108.

[35] Kitagbi, P. 2002. "Targeting Neurotensin Receptors with Agonists and Antagonists for Therapeutic Purposes." Current Opinion in Drug Discovery and Development 5 (5): 764-76.

[36] Petrie, K. A., Schmidt, D., Bubser, M., Fadel, J., Carraway, R. E., and Deutch, A. Y. 2005. "Neurotensin Activates GABAergic Interneurons in the Prefrontal Cortex." Journal of Neuroscience 25 (7): 1629-36.

[37] Li, Z., Boules, M., Williams, K., Peris, J., and Richelsona, E. 2010. "The Novel Neurotensin Analog NT69L Blocks Phencyclidine (PCP)-Induced Increases in Locomotor Activity and PCP-Induced Increases in Monoamine and Amino Acids Levels in the Medial Prefrontal Cortex." Brain Research 1311: 28-36.

[38] Matthe, A. A. 2011. "Neuropeptides and Eletroconvulsive Treatment." Journal of Electroconvulsive Therapy 15 (1): 60-75.

[39] Malherbe, P., Knoflach, F., Hernandez, M. C., Hoffmann, T., Schnider, P., Porter, R. H., Wettstein, J. G., Ballard, T. M., Spooren, W., and Steward, L. 2011. "Characterization of RO4583298 as a Novel, Potent, Dual Antagonist with in vivo Activity at Tachykinin NK1 and NK3 Receptors." British Journal of Pharmacology 162 


\section{How to Improve the Therapeutic Effect of the Antipsychotic Drugs}

(4): 929-46.

[40] Werkman, T. R., McCreary, A. C., Kruse, C. G., and Wadman, W. J. 2011. "NK3 Receptors Mediate an Increase in Firing Rate of Midbrain Dopamine Neurons of the Rat and the Guinea Pig." Synapse 65 (8): 814-26.

[41] Harrison, P. J., and Weinberger, D. R. 2005. "Schizophrenia Genes, Gene Expression, and Neuropathology: On Matter of Their Consequence." Molecular Psychiatry 10 (1): 40-68.

[42] Clinton, S. M., Haroutunian, V., Davis, K. L., and Meador-Woodruff, J. H. 2003. "Altered Transcript Expression of NMDA Receptor-Associated Postsynaptic Proteins in the Thalamus of Subjects with Schizophrenia." American Journal of Psychiatry 160 (6): 1100-9.

[43] Talbot, K., Eidem, W. L., Tinsley, C. L., Benson, M. A., Thompson, E. W., Smith, R. J., Hahn, C. G., Siegel, S. J., Trojanowski, J. Q., Gur, R. E., Blake, D. J., and Arnold, S. E. 2004. "Dysbindin-1 Is Reduced in Intrinsic, Glutaminergic Terminals of the Hippocampal Formation in Schizophrenia." Journal of Clinical Investigation 113 (9): 1353-63.

[44] Fatjó-Vilas, M., Papiol, S., Estrada, G., Bombín, I., Peralta, V., Rosa, A., Parellada, M., Miret, S., Martín, M., Lázaro, L., Campanera, S., Muñoz, M. J., Lera-Miguel, S., Arias, B., Navarro, M. E., Castro-Fornieles, J., Cuesta, M. J., Arango, C., and Fañanás, L. 2011. "Dysbindin-1 Gene Contributes Differentially to Early- and Adult-Onset Forms of Functional Psychosis." American Journal of
Medical Genetics. Part B: Neuropsychiatric Genetics 156 (3): 322-33.

[45] Wei, Y. L., Li, C. X., Li, S. B., Liu, Y., and Hu, L. 2011. "Association Study of Monoamine Oxidase A/B Genes and Schizophrenia in Han Chinese." Behavioral and Brain Functions 7: 42.

[46] Costa, E., Grayson, D. R., Mitchell, C. P., Tremolizzo, L., Veldic, M., and Guidotti, A. 2003. "GABAergic Cortical Neuron Chromatin as a Putative Target to Treat Schizophrenia Vulnerability." Critical Reviews in Neurobiology 15 (2): 121-42.

[47] Hashimoto, T., Volk, D. W., Eggan, S. M., Mirnics, K., Pierri, J. N., Sun, Z., Sampson, A. R., and Lewis, D. A. 2003. "Gene Expression Deficits in a Subclass of GABA Neurons in the Prefrontal Cortex of Subjects with Schizophrenia." Journal of Neuroscience 23 (15): 6315-26.

[48] Thomas, S. P., Nandhra, H. S., and Singh, S. P. 2012. "Pharmacological Treatment of First-episode Schizophrenia: A Review of the Literature." Primary Care Companion to CNS Disorders 14 (1): pii. PCC, 11r011198.

[49] Elsworth, J. D., Groman, S. M., Jentsch, J. D., Valles, R., Shahid, M., Wong, E., Marston, H., and Roth, R. H. 2011. "Asenapine Effects on Cognitive and Monamine Dysfunction Elicited by Subchronic Phencyclidine Administration." Neuropharmacology 62 (3): 1442-52.

[50] Guay, D. R. 2011. "Comment on the Potential Utility of the New Atypical Antipsychotic Lurasidone in the Geriatric Population." The Consultant Pharmacy 26 (8): 579-82. 